\title{
Mapeamento da produção científica sobre os fatores de risco para atraso no desenvolvimento neuromotor infantil
}

The mapping of the scientific production about risk factors for delays in child neuromotor development

\author{
Mapeo de la producción científica sobre los factores de riesgo para el retraso en el desarrollo \\ neuromotor infantil
}

\begin{abstract}
Resumo
O estudo teve por objetivo mapear a produção científica sobre os fatores de risco para atraso no desenvolvimento neuromotor infantil. Trata-se de um estudo de revisão, utilizando análise bibliométrica, buscando mensurar os índices de produção e disseminação do conhecimento científico, sobre os fatores de risco para atraso no desenvolvimento neuromotor infantil. A busca dos artigos foi realizada nas bases de dados PubMed, SciELO, PEDro, LILACS, utilizando os descritores: fatores de risco, desenvolvimento infantil, e, crianças, nas línguas portuguesa, inglesa e espanhola, considerando o período de 2015 a 2020. Inicialmente, foram encontrados 3121 artigos. Destes, foram incluídos no presente estudo 37 artigos. Os fatores de risco mais citados foram: baixa escolaridade materna, baixo nível socioeconômico familiar e pouca disponibilidade de recursos para a estimulação da criança. Dos artigos selecionados apenas 13 foram publicados em periódicos com fator de impacto, e, ainda, observou-se um declínio na produção científica acerca do tema com o passar dos anos. Os resultados indicam a necessidade de mais estudos, com rígidos critérios metodológico, para que possam ser publicados em revistas com bom fator de impacto, facilitando o alcance de um maior público, e, possibilitar a elaboração de políticas públicas de saúde. Vale ressaltar, que para elaboração de políticas públicas eficazes, buscando proporcionar o desenvolvimento infantil de forma integral, é fundamental que haja o embasamento técnico-científico sobre os fatores de risco que interferem nesse processo. Tal embasamento deve ser encontrado na literatura, em estudos de qualidade e confiabilidade.
\end{abstract}

Palavras-chave: Fatores de risco; Desenvolvimento infantil; Crianças.

\begin{abstract}
This study aimed to map the scientific production about risks of child neuromotor development delays. This is a revision study, using bibliometric analyses and seeking to measure the indexes of production and dissemination of scientific knowledge about risk factors for delays in child neuromotor development. Articles were searched in the databases PubMed, SciELO, PEDro, and LILACS, using the descriptors: risk factors, child development, and children. Papers selected were in Portuguese, English, and Spanish, published from 2015 to 2020. At first, 3121 articles were found. The study included 37 articles. The most cited risk factors were low educational level of the mother, low socioeconomic level of the family, and little available resources to stimulate the child. From the articles selected, only 13 had been published in journals with impact factors, and there was a reduction in the scientific
\end{abstract}


production on the topic throughout the years. The results suggest that further studies are necessary, with rigid methodological criteria, so they can be published in journals with good impact factors, which would make it easier for a larger public to access them and enable the elaboration of public health policies. It should also be mentioned that, to elaborate efficient public policies to promote integral child development, there must be technical-scientific knowledge regarding the risk factors that interfere with this process. This knowledge must be found in literature and in reliable quality studies.

Keywords: Risk factors; Child development; Children.

\section{Resumen}

El estudio tuvo como objetivo mapear la producción científica sobre los factores de riesgo para el retraso en el desarrollo neuromotor infantil. Se trató de un estudio de revisión, utilizando el análisis bibliométrico buscando medir los índices de producción y diseminación del conocimiento científico sobre los factores de riesgo para el retraso en el desarrollo neuromotor infantil. La búsqueda de artículos fue realizada en las bases de datos PubMed, SciELO, PEDro, LILACS, utilizando los descriptores: factores de riesgo, desarrollo infantil y niños, en los idiomas portugués, inglés y español, considerando el periodo de 2015 a 2020. Inicialmente fueron encontrados 3121 artículos. Fueron incluidos en el presente estudio 37 artículos. Los factores de riesgo más citados fueron: baja escolaridad materna, bajo nivel socioeconómico familiar y poca disponibilidad de recursos para la estimulación del niño. De los artículos seleccionados apenas 13 fueron publicados en revistas con factor de impacto y, además, se observó una disminución en la producción científica acerca del tema con el paso de los años. Los resultados indican la necesidad de más estudios, con rígidos criterios metodológicos, para que puedan ser publicados en revistas con buen factor de impacto facilitando el alcance de un mayor publico y posibilitar la elaboración de políticas publicas de salud. Cabe resaltar que para la elaboración de políticas publicas eficaces, buscando proporcionar el desarrollo infantil de forma integral, es fundamental que haya un fundamento técnico-científico sobre los factores de riesgo que intervienen en este proceso. Tal fundamento debe ser encontrado en la literatura en estudios de calidad y confiabilidad.

Palabras clave: Factores de riesgo; Desarrollo infantil; Niños.

\section{Introdução}

O desenvolvimento é um processo qualitativo contínuo, composto pela aquisição das habilidades humanas desenvolvidas progressivamente durante a vida (Torquato, Collet, Forte, França, Silva, \& Reichert, 2019). Este está relacionado com a maturação do sistema nervoso central, e se divide em quatro grandes domínios: motor grosseiro, motor fino, social e linguístico. Com o desaparecimento dos reflexos primitivos a criança desenvolve habilidades nesses quatro domínios e alcança os marcos do desenvolvimento. No entanto, cada indivíduo possui seu próprio ritmo para se desenvolver, o que é considerado normal, desde que não haja desvio significativo da sequência esperada (Silva, Cavalcante, Heumann, \& Lima, 2016). Ainda neste sentido, sabe-se por evidências científicas, que fatores físicos, biológicos, sociais e ambientais desfavoráveis podem gerar prejuízos a longo prazo no desenvolvimento (Martins, Vilela, Pereira, \& Cária, 2013; Silva, Engstron, \& Miranda, 2015).

A primeira infância é a fase crucial do desenvolvimento humano, pois, entre o período pré-natal e os anos iniciais da infância, é que ocorre grande parte do desenvolvimento cerebral. No entanto, fatores intrínsecos relacionados a herança genética e fatores biológicos, bem como, fatores extrínsecos provenientes do ambiente, interferem diretamente neste processo (Souza, \& Veríssimo, 2015). Como fatores de risco intrínsecos são considerados: idade gestacional, peso ao nascimento, patologias neonatais ou deficiências físicas. Já como fatores extrínsecos são considerados: baixa escolaridade dos pais, residência em área de risco socioeconômico e/ou ambiente domiciliar pouco ou nada adequado. A influência direta desses fatores no desenvolvimento neuromotor, foi observada em crianças de zero a 30 meses de idade, com história clínica de prematuridade, diagnóstico clínico de desnutrição moderada a grave, apresentando correlação entre baixa escolaridade materna, relação monoparental e intercorrências neonatais. O domínio do desenvolvimento mais acometido foi a linguagem, seguida pela motricidade fina. Além disso, observou-se que o ambiente em que a criança vive, principalmente, em relação a receptividade dos pais influencia o desenvolvimento típico (Zago, Pinto, Leite, Santos, \& Morais, 2017).

No estudo de Pereira, Valentini e Saccani (2016), com 100 lactentes que frequentavam creches públicas, por meio da Alberta Infant Motor Scale, Escala Bayley, Home Environment for Motor Development - Infant Scale, e Daily Activities of 
Infants Scale respectivamente, observou-se relação entre o desempenho motor e cognitivo do lactente, conhecimentos e práticas maternas, ambiente doméstico e fatores biológicos e socioeconômicos da criança. Os lactentes aumentaram o repertório motor e cognitivo ao longo do tempo, apresentando correlação direta entre desempenho motor, práticas e conhecimentos maternos, fatores biológicos e ambiente doméstico, contribuindo significativamente para o desenvolvimento motor e cognitivo infantil.

Desta forma, compreender os fatores de risco que influenciam o desenvolvimento em diferentes populações é fundamental para a construção das políticas públicas da primeira infância, com base em evidências científicas.

Considerando tal importância, o objetivo deste estudo foi realizar uma análise bibliométrica da literatura, a fim de mapear o estado da arte da produção científica atual, publicada entre 2015 e 2020, sobre os fatores de risco para atraso no desenvolvimento neuromotor infantil.

\section{Metodologia}

Trata-se de um estudo utilizando a técnica de análise bibliométrica, a qual permite mensurar os índices de produção e disseminação do conhecimento científico. A bibliometria busca analisar a informação, numa abordagem quantitativa, organizando e classificando características das publicações como os mecanismos de busca e sua forma de recuperação, permitindo conhecer o estado da arte em determinada área (Souza, Silva, \& Carvalho, 2010). Inicialmente, definiu-se os critérios de elegibilidade, sendo os seguintes: artigos de revisão sistemática, ensaio clínico e estudo de coorte, publicados entre os anos de 2015 e 2020, sobre os fatores que influenciam o desenvolvimento neurossensoriomotor infantil. Como critérios de não inclusão e exclusão considerou-se: artigos de opinião, série de casos, comunicações, estudos com modelos animais, e, os artigos que não estavam disponíveis na íntegra, com acesso livre. O levantamento bibliográfico foi realizado nas bases de dados National Library of Medicine (PubMed), Scientific Electronic Library Online (SciELO), Physiotherapy Evidence Database (PEDro), Literatura Latino-Americana e do Caribe em Ciências da Saúde (LILACS), com os descritores: fatores de risco, desenvolvimento infantil e crianças, nas línguas portuguesa, inglesa e espanhola. Após a seleção prévia dos estudos, foram excluídos os artigos duplicados nas bases de dados, e, então, feita a leitura dos resumos dos estudos restantes, buscando verificar se atendiam os critérios de elegibilidade. Na sequência, os estudos foram lidos na íntegra e incluídos os que atenderam os critérios de inclusão.

Para a análise bibliométrica e caracterização do estado da arte, ou seja, o rigor metodológico dos estudos selecionados, foram extraídos e tabulados os seguintes dados: número de artigos publicados na revista, país de publicação, fator de impacto da revista, ano de publicação, palavras chaves, autores, número de citações, delineamento da pesquisa, número de participantes da pesquisa, e resultados.

\section{Resultados}

A busca com os descritores predeterminados, resultou na identificação de 3121 artigos, dos quais foram excluídos 3077 após leitura do título/resumo, restando 44 artigos. Uma nova filtragem foi realizada, e mais sete artigos foram excluídos por estarem duplicados nas bases de dados. Assim, a amostra final foi composta por 37 artigos, os quais foram analisados na íntegra. A base de dados com maior número de publicações acerca do tema pesquisado foi a PubMed correspondendo a $85 \%$ do total, seguida pela LILACS com 8\%, e, por último, a SciELO com 6\%. A Figura 1 demonstra o percurso seguido e as bases de dados consultadas para seleção dos artigos incluídos neste estudo. 
Figura 1. Fluxograma de seleção dos artigos.

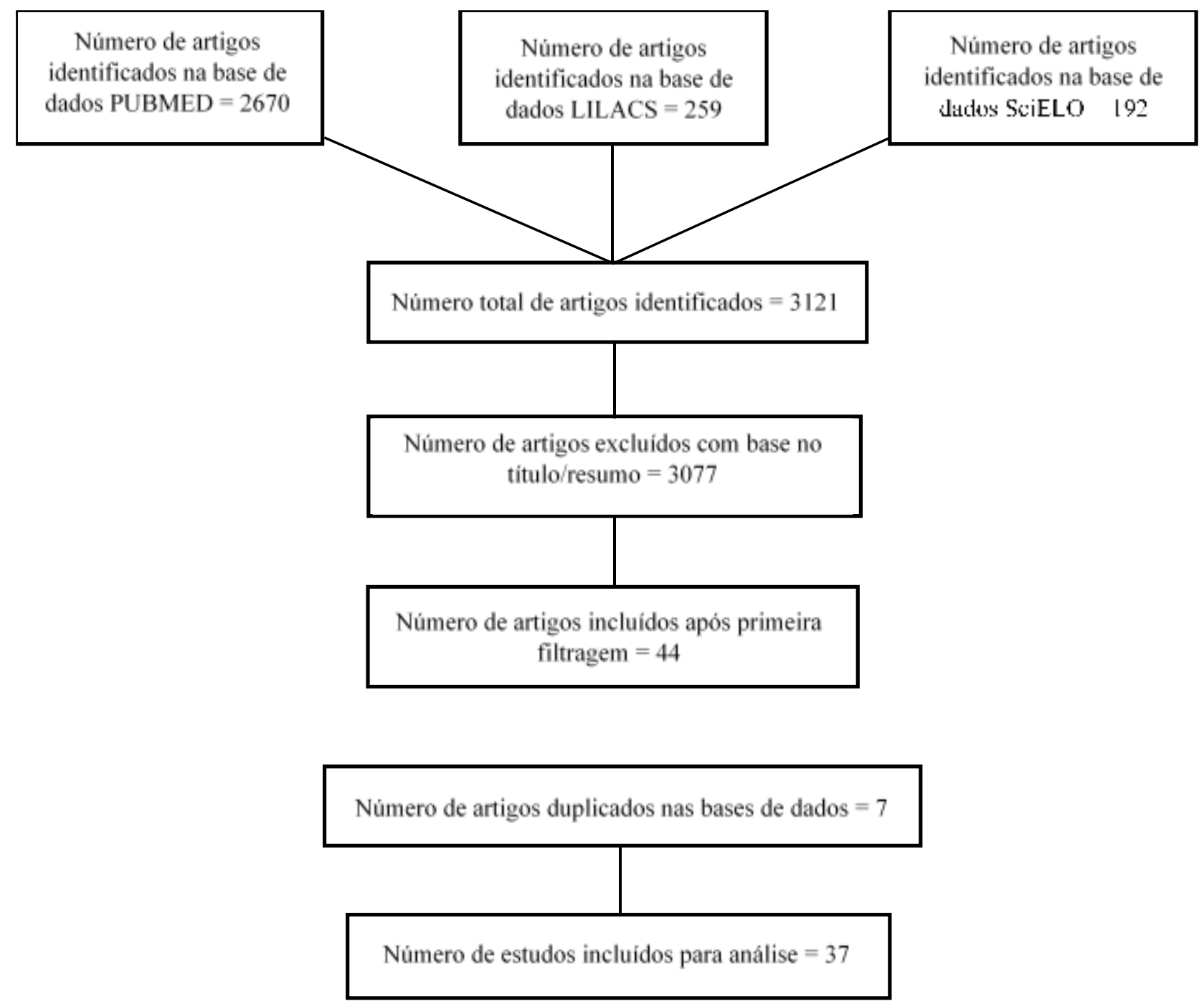

Fonte: Autores. 
Tabela 1. Caracterização das publicações incluídas no estudo.

\begin{tabular}{|c|c|c|c|c|c|c|c|}
\hline Título & Autores & Citações & $\mathbf{N}$ & $\begin{array}{l}\text { Tipo de participantes/Local do } \\
\text { estudo }\end{array}$ & $\begin{array}{l}\text { Delineamento } \\
\text { da pesquisa }\end{array}$ & Resultados & $\begin{array}{l}\text { Fator de } \\
\text { impacto }\end{array}$ \\
\hline $\begin{array}{l}\text { Association between } \\
\text { neuropsychomotor } \\
\text { development and } \\
\text { biological } \\
\text { and environmental risk } \\
\text { factors in early childhood } \\
\text { children }\end{array}$ & $\begin{array}{l}\text { Jéssica Teixeira de Carvalho } \\
\text { Zago, Priscilla Avelino } \\
\text { Ferreira Pinto, Hércules } \\
\text { Ribeiro Leite, Juliana Nunes } \\
\text { Santos, Rosane Luzia de Souza } \\
\text { Morais. }\end{array}$ & 15 & 30 & $\begin{array}{l}\text { A população do estudo foi constituída } \\
\text { por todos os prontuários de crianças } \\
\text { de } 0 \text { a } 30 \text { meses de idade, atendidas } \\
\text { pelo Centro Viva Vida de Referência } \\
\text { Secundária CVVRS, com história } \\
\text { clínica de prematuridade e/ou } \\
\text { diagnóstico clínico de desnutrição } \\
\text { moderada à grave/ Diamantina, Brasil }\end{array}$ & $\begin{array}{l}\text { Estudo } \\
\text { transversal }\end{array}$ & $\begin{array}{l}\text { O principal domínio afetado foi o da linguagem. Maior } \\
\text { escolaridade materna e constituição familiar biparental } \\
\text { demonstraram associação com o adequado } \\
\text { desenvolvimento infantil. As crianças com menor } \\
\text { desempenho nos testes, apresentaram intercorrências } \\
\text { neonatais e necessitaram de internação no centro de } \\
\text { terapia intensiva. A menor receptividade dos pais e } \\
\text { disponibilidade de materiais para aprendizagem no } \\
\text { domicílio foram fatores associados ao pior } \\
\text { desempenho das crianças no teste de triagem Denver } \\
\text { II. }\end{array}$ & - \\
\hline $\begin{array}{l}\text { Evaluation of child motor } \\
\text { development and its } \\
\text { association with social } \\
\text { vulnerability }\end{array}$ & $\begin{array}{l}\text { Daiane Alves Delgado, Rita } \\
\text { Cassiana Michelon, Laís } \\
\text { Rodrigues Gerzson, Carla } \\
\text { Skilhan de Almeida, Maria da } \\
\text { Graça Alexandre. }\end{array}$ & 02 & 110 & $\begin{array}{l}\text { Crianças entre quatro e } 17 \text { meses, } \\
\text { clinicamente estáveis, sem suporte de } \\
\text { oxigênio e com alta breve prevista/ } \\
\text { Porto Alegre, Brasil }\end{array}$ & $\begin{array}{l}\text { Estudo } \\
\text { transversal, } \\
\text { descritivo, com } \\
\text { amostra não } \\
\text { probabilística por } \\
\text { conveniência. }\end{array}$ & $\begin{array}{l}\text { Déficits no desenvolvimento motor foi relacionado } \\
\text { com significância estatística à vacinas atrasadas, } \\
\text { convivência com tabagistas em casa e recebimento de } \\
\text { benefício socioeconômico. }\end{array}$ & - \\
\hline $\begin{array}{l}\text { Socioeconomic } \\
\text { diversities and infant } \\
\text { development at } 6 \text { to } 9 \\
\text { months in a poverty area } \\
\text { of São Paulo, Brazil }\end{array}$ & $\begin{array}{l}\text { Patricia Tella, Luciane da Rosa } \\
\text { Piccolo, Mayra Lemus Rangel, } \\
\text { Luis Augusto Rohde, } \\
\text { Guilherme Vanoni Polanczyk, } \\
\text { Euripides Constantino Miguel, } \\
\text { Sandra Josefina Ferraz Ellero } \\
\text { Grisi, Bacy Fleitlich-Bilyk, } \\
\text { Alexandre Archanjo Ferraro }\end{array}$ & 06 & 444 & $\begin{array}{l}\text { Crianças entre } 6 \text { e } 9 \text { meses de idade } \\
\text { selecionados em um bairro de baixo } \\
\text { nível socioeconômico na zona oeste/ } \\
\text { São Paulo, Brasil }\end{array}$ & $\begin{array}{l}\text { Estudo } \\
\text { transversal }\end{array}$ & $\begin{array}{l}\text { Observou-se associação positiva entre a situação } \\
\text { socioeconômica e o desempenho motor e da } \\
\text { linguagem. Além disso, verificou-se que quanto maior } \\
\text { o nível de escolaridade materna melhor o desempenho } \\
\text { na linguagem e escores cognitivos do bebê. }\end{array}$ & - \\
\hline $\begin{array}{l}\text { Effect of school type and } \\
\text { family income on motor } \\
\text { competence changes in } \\
\text { pre-school children: a } \\
\text { repeated cross sectional }\end{array}$ & $\begin{array}{l}\text { Daniel da Rocha Queiroz, } \\
\text { Túlio Guilherme Martins } \\
\text { Guimarães, Carolina Maria } \\
\text { Coelho Campos, Anderson } \\
\text { Henry Pereira Feitoza, Rafael }\end{array}$ & 0 & 552 & $\begin{array}{l}\text { Crianças com idade entre } 3 \text { e } 5 \text { anos } \\
\text { de } 28 \text { escolas públicas ou particulares/ } \\
\text { Recife, Brasil }\end{array}$ & $\begin{array}{l}\text { Estudo } \\
\text { transversal }\end{array}$ & $\begin{array}{l}\text { O tipo de escola e renda aumentaram o tamanho do } \\
\text { efeito nas diferenças das habilidades de controle de } \\
\text { objetos e no quociente motor geral. }\end{array}$ & - \\
\hline
\end{tabular}


Research, Society and Development, v. 10, n. 10, e411101018881, 2021

(CC BY 4.0) | ISSN 2525-3409 | DOI: http://dx.doi.org/10.33448/rsd-v10i10.18881

\begin{tabular}{|c|c|c|c|c|c|c|c|}
\hline study & $\begin{array}{l}\text { dos Santos Henrique, Marcos } \\
\text { André Moura dos Santos and } \\
\text { Maria Teresa Cattuzzo }\end{array}$ & & & & & & \\
\hline $\begin{array}{l}\text { Influence of congenital } \\
\text { heart disease on the } \\
\text { neuropsychomotor } \\
\text { development of infants }\end{array}$ & $\begin{array}{l}\text { Ítalo Ribeiro Paula, Janaína } \\
\text { Carla Silva Oliveira, Ana } \\
\text { Carolina Ferreira Batista, } \\
\text { Lizandra Caroline Santana } \\
\text { Nascimento, Lúcio Borges de } \\
\text { Araújo, Márcia Berbert } \\
\text { Ferreira, Miria Benincasa } \\
\text { Gomes, Vivian Mara } \\
\text { Gonçalves de Oliveira } \\
\text { Azevedo }\end{array}$ & 0 & 18 & $\begin{array}{l}\text { Foram incluídos os lactentes com } \\
\text { diagnóstico de cardiopatias } \\
\text { congênitas cianogênicas e } \\
\text { acianogênicas, com idade entre um e } \\
18 \text { meses, que estavam em } \\
\text { acompanhamento ambulatorial/ } \\
\text { Uberlândia, Brasil }\end{array}$ & $\begin{array}{l}\text { Estudo } \\
\text { observacional } \\
\text { transversal }\end{array}$ & $\begin{array}{l}\text { Houve correlação das escalas do BSID-III com as } \\
\text { variáveis quantitativas analisadas: escala motora com } \\
\text { o peso e com uso de oxigenoterapia, já para as } \\
\text { variáveis qualitativas as associações foram entre: } \\
\text { escala motora e condição socioeconômica, escala } \\
\text { motora e comunicação interatrial e escala da } \\
\text { linguagem e comunicação interatrial. }\end{array}$ & - \\
\hline $\begin{array}{l}\text { The influence of } \\
\text { socioeconomic status on } \\
\text { gross motor skills in } \\
\text { school-age children }\end{array}$ & $\begin{array}{l}\text { Giselle Bernardo Candéa, } \\
\text { M.Sc., Julio Guilherme Silva, } \\
\text { D.Sc., André Luis Peixoto } \\
\text { Candéa, D.Sc., Jéssica de } \\
\text { Medeiros Vidal, Silvio de } \\
\text { Cassio Costa Telles, D.Sc. }\end{array}$ & 02 & 41.497 & $\begin{array}{l}\text { Artigos relacionados à influência do } \\
\text { status socioeconômico sobre as } \\
\text { habilidades motoras grossas em } \\
\text { crianças em idade escolar nas bases } \\
\text { de dados Scopus e Pubmed/ Rio de } \\
\text { Janeiro, Brasil }\end{array}$ & $\begin{array}{l}\text { Revisão } \\
\text { sistemática }\end{array}$ & $\begin{array}{l}\text { Os principais resultados mostraram uma prevalência } \\
\text { de crianças de alto nível socioeconômico com maior } \\
\text { pontuação nas habilidades motoras em relação às } \\
\text { crianças de baixo nível socioeconômico. }\end{array}$ & - \\
\hline $\begin{array}{l}\text { Biopsychosocial factors } \\
\text { contributing to delayed } \\
\text { motor development in } \\
\text { children: a longitudinal } \\
\text { study }\end{array}$ & $\begin{array}{l}\text { Ana Paula Maurilia dos } \\
\text { Santos, Lorena Natal } \\
\text { Villaverde, Antônia Natália } \\
\text { Ferreira Costa, Manoella de } \\
\text { Oliveira Santos, Elaine } \\
\text { Cristina Gregório, Lucia Maria } \\
\text { Andreis, Francisco Rosa Neto }\end{array}$ & 06 & 17 & $\begin{array}{l}\text { Escolares matriculados no quarto e } \\
\text { quinto ano do Ensino Fundamental de } \\
14 \text { escolas públicas/ Florianópolis, } \\
\text { Brasil }\end{array}$ & $\begin{array}{l}\text { Estudo } \\
\text { longitudinal }\end{array}$ & $\begin{array}{l}\text { Fatores de risco biológico e ambientais, como } \\
\text { dificuldades na aprendizagem escolar e baixo nível } \\
\text { socioeconômico foram relacionados ao atraso no } \\
\text { desenvolvimento motor. }\end{array}$ & - \\
\hline $\begin{array}{l}\text { Assessment of Motor } \\
\text { Development of Children } \\
\text { with Congenital Heart } \\
\text { Disease }\end{array}$ & $\begin{array}{l}\text { Lais Sena Leal, Rafael Luiz } \\
\text { Morais da Silva, Karla Maria } \\
\text { Siqueira Coelho Aita, Rogéria } \\
\text { Pimentel de Araújo Monteiro, } \\
\text { Tatiana Carvalho de } \\
\text { Montalvão }\end{array}$ & 07 & 30 & $\begin{array}{l}\text { Crianças, de ambos os sexos, } \\
\text { portadoras de cardiopatia congênita, } \\
\text { em cuidados ambulatoriais/ Belém, } \\
\text { Brasil }\end{array}$ & $\begin{array}{l}\text { Estudo } \\
\text { transversal } \\
\text { quantitativo }\end{array}$ & $\begin{array}{l}\text { As habilidades motoras avaliadas encontravam-se } \\
\text { significativamente abaixo do nível normal médio. Três } \\
\text { aspectos do desenvolvimento motor revelaram } \\
\text { importante risco ao desenvolvimento: esquema } \\
\text { corporal, organização espacial e organização temporal. }\end{array}$ & - \\
\hline $\begin{array}{l}\text { Assessment of the } \\
\text { neuropsychomotor } \\
\text { development in the first } \\
\text { year of life of premature } \\
\text { infants with and without } \\
\text { bronchopulmonary } \\
\text { dysplasia }\end{array}$ & $\begin{array}{l}\text { Letycia Vieira Silva, Lúcio } \\
\text { Borges de Araújo, Vivian } \\
\text { Mara Gonçalves de Oliveira } \\
\text { Azevedo }\end{array}$ & 09 & 79 & $\begin{array}{l}\text { Lactentes prematuros, com peso } \\
\text { menor que } 1.500 \mathrm{~g} \text { ao nascer e } \\
\text { diagnóstico de displasia } \\
\text { broncopulmonar na idade corrigida de } \\
6 \text { e } 9 \text { meses/ Uberlândia, Brasil }\end{array}$ & $\begin{array}{l}\text { Estudo } \\
\text { transversal } \\
\text { retrospectivo }\end{array}$ & $\begin{array}{l}\text { Os lactentes com displasia broncopulmonar } \\
\text { apresentaram maior atraso no desenvolvimento } \\
\text { neuropsicomotor quando comparados àqueles sem } \\
\text { displasia broncopulmonar. Os fatores associados com } \\
\text { maior incidência para alterações no desenvolvimento } \\
\text { neuropsicomotor, além da displasia broncopulmonar, } \\
\text { foram: esteroide antenatal, sexo, peso ao nascimento, } \\
\text { escore de Apgar no quinto minuto, Score for Neonatal } \\
\text { Acute Physiology with Perinatal Extension, tempo de }\end{array}$ & - \\
\hline
\end{tabular}


Research, Society and Development, v. 10, n. 10, e411101018881, 2021

(CC BY 4.0) | ISSN 2525-3409 | DOI: http://dx.doi.org/10.33448/rsd-v10i10.18881

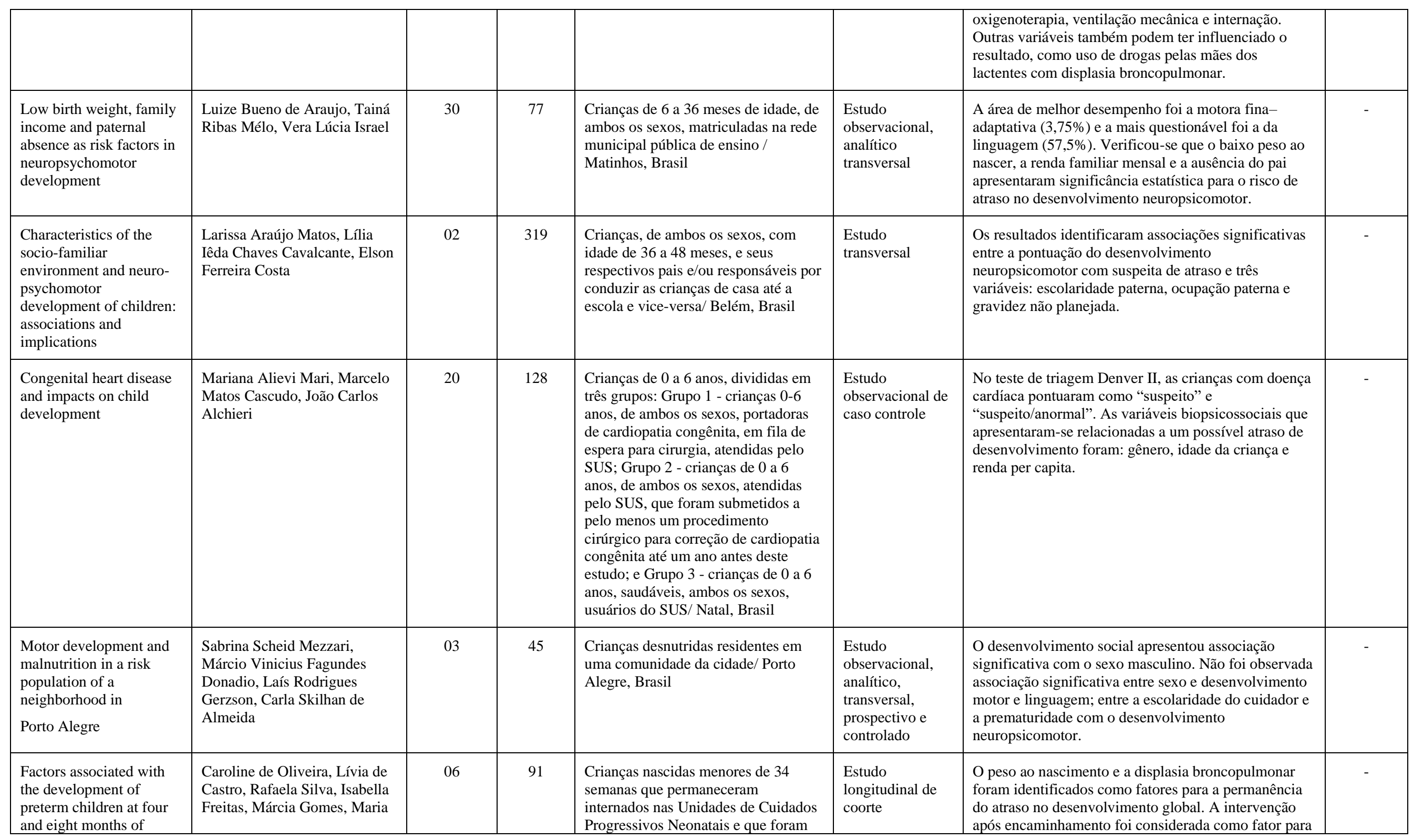


Research, Society and Development, v. 10, n. 10, e411101018881, 2021

(CC BY 4.0) | ISSN 2525-3409 | DOI: http://dx.doi.org/10.33448/rsd-v10i10.18881

\begin{tabular}{|c|c|c|c|c|c|c|c|}
\hline corrected gestational age & Cândida & & & $\begin{array}{l}\text { acompanhadas em ambulatório/ Belo } \\
\text { Horizonte, Brasil }\end{array}$ & prospectivo & a melhora no desenvolvimento. & \\
\hline $\begin{array}{l}\text { Factors associated with } \\
\text { neurodevelopment in } \\
\text { children 6-18 months of } \\
\text { age in public daycare } \\
\text { centers in João Pessoa, } \\
\text { Paraíba State, Brazil }\end{array}$ & $\begin{array}{l}\text { Ângela Cristina Dornelas da } \\
\text { Silva, Elyne Montenegro } \\
\text { Engstron, Cláudio Torres de } \\
\text { Miranda }\end{array}$ & 38 & 112 & $\begin{array}{l}\text { Crianças, com idade entre } 6 \text { e } 18 \\
\text { meses, de ambos os sexos, } \\
\text { frequentadoras de berçários em dez } \\
\text { creches, e suas respectivas mães/ João } \\
\text { Pessoa, Brasil }\end{array}$ & Estudo seccional & $\begin{array}{l}52,7 \% \text { das crianças apresentaram alteração no } \\
\text { desenvolvimento infantil. A alteração no } \\
\text { desenvolvimento infantil foi correlacionada aos } \\
\text { seguintes fatores: a criança ter mais que } 12 \text { meses de } \\
\text { idade, a criança ter nascido de parto vaginal, } \\
\text { necessidade de fototerapia após o nascimento e a } \\
\text { creche não ter apoio da Estratégia Saúde da Família. }\end{array}$ & - \\
\hline $\begin{array}{l}\text { Developmental delays } \\
\text { among children from day } \\
\text { care center of } \\
\text { socially disadvantaged } \\
\text { families }\end{array}$ & $\begin{array}{l}\text { Maria Cristina Triguero Veloz } \\
\text { Teixeira, Felipe Alckmin- } \\
\text { Carvalho, Deisy Ribas } \\
\text { Emerich, Priscilla Veloz } \\
\text { Cevallos, Cristiane Silvestre de } \\
\text { Paula }\end{array}$ & 0 & 100 & $\begin{array}{l}\text { Crianças com idade entre } 16 \text { e } 24 \\
\text { meses, regularmente matriculadas e } \\
\text { frequentando creches públicas/ } \\
\text { Barueri, Brasil }\end{array}$ & $\begin{array}{l}\text { Estudo } \\
\text { transversal }\end{array}$ & $\begin{array}{l}\text { O domínio de maior prejuízo foi o da linguagem, } \\
\text { seguido pelo domínio de cuidado pessoal-social. Os } \\
\text { resultados do estudo apontam para a necessidade de } \\
\text { incluir na avaliação do desenvolvimento infantil } \\
\text { fatores familiares e educacionais, comumente } \\
\text { associados a atrasos de desenvolvimento. }\end{array}$ & - \\
\hline $\begin{array}{l}\text { Investigation of risk } \\
\text { factors for motor } \\
\text { development of infants } \\
\text { up to three month old }\end{array}$ & $\begin{array}{l}\text { Veronica Aparecida Pereira, } \\
\text { Carla Suzana Oliveira e Silva- } \\
\text { Marinho, Olga Maria Piazentin } \\
\text { Rolim Rodrigues, Taís } \\
\text { Chiodelli, Millena Lima } \\
\text { Donatto }\end{array}$ & 04 & 57 & $\begin{array}{l}\text { Mães que participaram de um } \\
\text { programa de estimulação precoce/ } \\
\text { Dourados, Brasil }\end{array}$ & $\begin{array}{l}\text { Estudo descritivo } \\
\text { e correlacional }\end{array}$ & $\begin{array}{l}\text { Quando considerada idade das mães, observou-se um } \\
\text { maior percentual de partos prematuros entre as mães } \\
\text { adolescentes ou com mais de } 35 \text { anos, apresentando } \\
\text { uma tendência da variável idade materna como fator } \\
\text { de risco para prematuridade. }\end{array}$ & - \\
\hline $\begin{array}{l}\text { Impact of extrinsic } \\
\text { factors on fine motor } \\
\text { performance of children } \\
\text { attending day care }\end{array}$ & $\begin{array}{l}\text { Carolina Corsi, Mariana } \\
\text { Martins dos Santos, Luísa de } \\
\text { Andrade Perez Marques, Nelci } \\
\text { Adriana Cicuto Ferreira Rocha }\end{array}$ & 08 & 94 & $\begin{array}{l}\text { Crianças, na faixa de dois anos, de } \\
\text { ambos os sexos, de creches públicas e } \\
\text { de creches particulares/ São Carlos, } \\
\text { Brasil }\end{array}$ & $\begin{array}{l}\text { Estudo } \\
\text { transversal }\end{array}$ & $\begin{array}{l}\text { O tempo de creche apresentou correlação positiva com } \\
\text { o desempenho das crianças em algumas tarefas de } \\
\text { motricidade fina, demonstrando que as atividades } \\
\text { desenvolvidas nas creches foram importantes para o } \\
\text { refinamento de habilidades motoras específicas, } \\
\text { enquanto o desempenho motor fino geral foi } \\
\text { relacionado com a escolaridade materna e com o } \\
\text { subitem da escala ITERS-R "Linguagem e } \\
\text { compreensão". }\end{array}$ & - \\
\hline $\begin{array}{l}\text { Developmental in } \\
\text { children aged } 0-5 \text { years } \\
\text { living under unfavourable } \\
\text { environmental conditions }\end{array}$ & $\begin{array}{l}\text { Horacio Lejarragaa, Diana M. } \\
\text { Kelmanskyb, Fernando } \\
\text { Nunesc }\end{array}$ & 0 & 5401 & $\begin{array}{l}\text { Crianças menores de } 6 \text { anos de idade } \\
\text { de áreas com uma alta proporção de } \\
\text { população com necessidades básicas } \\
\text { insatisfeitas/ Florencio Varela e Bacia } \\
\text { Matanza, Argentina }\end{array}$ & $\begin{array}{l}\text { Estudo } \\
\text { transversal }\end{array}$ & $\begin{array}{l}\text { A fase normal de desenvolvimento foi atribuída ao } \\
\text { efeito protetor da mãe e do ambiente familiar; a fase } \\
\text { de atraso progressivo, à influência desfavorável do } \\
\text { ambiente, e a fase de recuperação parcial, ao } \\
\text { atendimento na creche. }\end{array}$ & - \\
\hline $\begin{array}{l}\text { Child development in } \\
\text { poor areas of Peru }\end{array}$ & $\begin{array}{l}\text { Adrián Alberto Díaz, Jorge } \\
\text { Bacallao Gallestey, Rocío } \\
\text { Vargas-Machuca, Roxana } \\
\text { Aguilar Velarde }\end{array}$ & 16 & 1176 & $\begin{array}{l}\text { Famílias com crianças menores de } 5 \\
\text { anos, residentes em sete províncias/ } \\
\text { Apurímac, Ayacucho, Huancavelica e } \\
\text { Loreto, Peru }\end{array}$ & $\begin{array}{l}\text { Estudo } \\
\text { transversal } \\
\text { descritivo }\end{array}$ & $\begin{array}{l}\text { As crianças da zona rural, filhos de mães com baixa } \\
\text { escolaridade e pertencentes a } \\
\text { domicílios com necessidades básicas insatisfatórias, } \\
\text { apresentaram pontuação mais baixa na avaliação do }\end{array}$ & - \\
\hline
\end{tabular}


Research, Society and Development, v. 10, n. 10, e411101018881, 2021

(CC BY 4.0) | ISSN 2525-3409 | DOI: http://dx.doi.org/10.33448/rsd-v10i10.18881

\begin{tabular}{|c|c|c|c|c|c|c|c|}
\hline & & & & & & desenvolvimento motor e da linguagem. & \\
\hline $\begin{array}{l}\text { Early child development } \\
\text { inequalities and } \\
\text { associated factors } \\
\text { between public } \\
\text { and private providers at } \\
\text { metropolitan region in } \\
\text { Chile }\end{array}$ & $\begin{array}{l}\text { Paula Bedregal, Viviana } \\
\text { Hernández, M. Verónica } \\
\text { Mingo, Carla Castanón, } \\
\text { Patricia Valenzuela, Rosario } \\
\text { Moore, Rolando de la Cruz, } \\
\text { Daniela Castro }\end{array}$ & 11 & 1045 & $\begin{array}{l}\text { Crianças de } 30 \text { a } 58 \text { meses, } \\
\text { acompanhadas nas redes pública e } \\
\text { privada de saúde/ Região } \\
\text { metropolitana, Chile }\end{array}$ & $\begin{array}{l}\text { Estudo } \\
\text { transversal }\end{array}$ & $\begin{array}{l}\text { Das crianças avaliadas } 14,4 \% \text { da rede privada e } 30,4 \% \\
\text { da rede pública tiveram desenvolvimento alterado. } \\
\text { Concluiu-se que, independentemente do nível } \\
\text { socioeconômico, são fatores de risco: Apgar menor } \\
\text { que } 7 \text { e portadores de doenças crônicas na infância. } \\
\text { Como fator protetor foi considerado a casa com } \\
\text { recursos para aprender e brincar. }\end{array}$ & - \\
\hline $\begin{array}{l}\text { Factors influencing } \\
\text { developmental delay } \\
\text { among young children in } \\
\text { poor rural China: a latent } \\
\text { variable approach }\end{array}$ & $\begin{array}{l}\text { Jingxu Zhang, Sufang Guo, } \\
\text { Ying Li, Qianwei Wei, } \\
\text { Cuihong Zhang, Xiaoli Wang, } \\
\text { Shusheng Luo, Chunxia Zhao, } \\
\text { Robert W Scherpbier }\end{array}$ & 10 & 2514 & $\begin{array}{l}\text { Crianças de } 6 \text { a } 35 \text { meses e seus } \\
\text { cuidadores principais/ Área rural, } \\
\text { China }\end{array}$ & $\begin{array}{l}\text { Estudo } \\
\text { transversal }\end{array}$ & $\begin{array}{l}\text { A prevalência de suspeita de atraso no } \\
\text { desenvolvimento } \\
\text { foi inversamente associado com a idade. Foi } \\
\text { demonstrado correlação direta entre o cuidado e } \\
\text { estímulo do cuidador e o nível de hemoglobina da } \\
\text { criança, e, correlação indireta entre os fatores } \\
\text { sociodemográficos do cuidador e a suspeita de atraso } \\
\text { no desenvolvimento. }\end{array}$ & - \\
\hline $\begin{array}{l}\text { Maternal posttraumatic } \\
\text { stress disorder and infant } \\
\text { developmental outcomes } \\
\text { in a South African birth } \\
\text { Cohort Study }\end{array}$ & $\begin{array}{l}\text { Nastassja Koen, Kirsty } \\
\text { Brittain, Kirsten A. Donald, } \\
\text { Whitney Barnett, Sheri } \\
\text { Koopowitz, Karen Maré, } \\
\text { Heather J. Zar, Dan J. Stein }\end{array}$ & 16 & 111 & $\begin{array}{l}\text { Mulheres grávidas com } 20-28 \\
\text { semanas de gestação em duas clínicas } \\
\text { de atenção primária e seus respectivos } \\
\text { filhos após o nascimento até os } 5 \text { anos } \\
\text { de idade/ Cape Town, África do Sul }\end{array}$ & Estudo de coorte & $\begin{array}{l}\text { O estresse pós-traumático materno foi } \\
\text { significativamente associado com o comportamento } \\
\text { motor fino e o comportamento adaptativo mais pobre } \\
\text { de seu filho; o último permanecendo significativo } \\
\text { quando ajustado para local, dependência de álcool e } \\
\text { escore do perímetro cefálico para a idade do bebê ao } \\
\text { nascer. }\end{array}$ & - \\
\hline $\begin{array}{l}\text { Maternal socio- } \\
\text { demographic and } \\
\text { psychological predictors } \\
\text { for risk of developmental } \\
\text { delays among young } \\
\text { children in Mongolia }\end{array}$ & $\begin{array}{l}\text { Amarjargal Dagvadorj, } \\
\text { Duurenbayar Ganbaatar, } \\
\text { Olukunmi O. Balogun, } \\
\text { Naohiro Yonemoto, } \\
\text { Bayasgalantai Bavuusuren, } \\
\text { Kenji Takehara, Rintaro Mori, } \\
\text { Moe Akahira-Azuma }\end{array}$ & 05 & 150 & $\begin{array}{l}\text { Crianças com idade entre } 13 \text { e } 24 \\
\text { meses/ Mongólia }\end{array}$ & $\begin{array}{l}\text { Estudo } \\
\text { longitudinal }\end{array}$ & $\begin{array}{l}\text { Observou-se associação negativa entre o risco de } \\
\text { atraso no desenvolvimento infantil e maior } \\
\text { escolaridade materna. Foram considerados também } \\
\text { preditores de atraso no desenvolvimento: idade } \\
\text { materna, sintomas de depressão materna, sexo } \\
\text { feminino da criança e mãe solteira. }\end{array}$ & 1.909 \\
\hline $\begin{array}{l}\text { Neurodevelopmental } \\
\text { outcome of late preterm } \\
\text { infants in Johannesburg, } \\
\text { South Africa }\end{array}$ & $\begin{array}{l}\text { Tanusha Ramdin, Daynia } \\
\text { Ballot, David Rakotsoane, } \\
\text { Lethile Madzudzo, Nicolette } \\
\text { Brown, Tobias Chirwa, Peter } \\
\text { Cooper, Victor Davies }\end{array}$ & 04 & 56 & $\begin{array}{l}\text { Recém nascidos prematuros com peso } \\
\text { menor que } 1500 \mathrm{~g} \text {, nascidos entre } 1 \text { de } \\
\text { julho de } 2013 \text { e } 30 \text { de junho de } 2014 \text {, } \\
\text { que necessitaram de internação em } \\
\text { unidade de terapia intensiva neonatal } \\
\text { e após a alta compareceram a pelo } \\
\text { menos uma visita de } \\
\text { acompanhamento à clínica do estudo/ } \\
\text { Joanesburgo, África do Sul }\end{array}$ & $\begin{array}{l}\text { Estudo de coorte } \\
\text { prospectivo }\end{array}$ & $\begin{array}{l}\text { Não houve diferença significativa no desempenho } \\
\text { cognitivo, na linguagem e motricidade entre bebês } \\
\text { prematuros tardios e controles. }\end{array}$ & 1.909 \\
\hline
\end{tabular}


Research, Society and Development, v. 10, n. 10, e411101018881, 2021

(CC BY 4.0) | ISSN 2525-3409 | DOI: http://dx.doi.org/10.33448/rsd-v10i10.18881

\begin{tabular}{|c|c|c|c|c|c|c|c|}
\hline $\begin{array}{l}\text { Nutritional, socio- } \\
\text { economic, and delivery } \\
\text { characteristics are } \\
\text { associated with } \\
\text { neurodevelopment in } \\
\text { tanzanian children }\end{array}$ & $\begin{array}{l}\text { Mia M. Blakstad, Emily R. } \\
\text { Smith, Analee Etheredge, } \\
\text { Lindsey M. Locks, Christine } \\
\text { M. McDonald, Roland Kupka, } \\
\text { Rodrick Kisenge, Said Aboud, } \\
\text { David Bellinger, Christopher } \\
\text { R. Sudfeld, Wafaie W. Fawzi, } \\
\text { Karim Manji, Christopher P. } \\
\text { Duggan }\end{array}$ & 03 & 453 & $\begin{array}{l}\text { Bebês tanzanianos, com } 15 \text { meses de } \\
\text { idade, expostos ao HIV, não } \\
\text { infectados e não expostos ao HIV que } \\
\text { foram inscritos em } 2 \text { ensaios } \\
\text { separados de micronutrientes/ } \\
\text { Tanzânia }\end{array}$ & $\begin{array}{l}\text { Estudo de coorte } \\
\text { prospectivo }\end{array}$ & $\begin{array}{l}\text { Entre os determinantes maternos, observou-se que a } \\
\text { baixa estatura materna previu os domínios cognitivo, } \\
\text { motor grosso, motor fino, linguagem expressiva e } \\
\text { linguagem receptiva da BSIDIII em crianças não } \\
\text { expostas ao HIV; baixa escolaridade materna previu } \\
\text { menor escore cognitivo e menor escore motor bruto } \\
\text { em crianças expostas ao HIV. }\end{array}$ & 2.029 \\
\hline $\begin{array}{l}\text { Predictors and pathways } \\
\text { of language and motor } \\
\text { development in four } \\
\text { prospective cohorts of } \\
\text { young children in Ghana, } \\
\text { Malawi, and Burkina } \\
\text { Faso }\end{array}$ & $\begin{array}{l}\text { Elizabeth L. Prado, Souheila } \\
\text { Abbeddou, Seth Adu- } \\
\text { Afarwuah, Mary Arimond, Per } \\
\text { Ashorn, Ulla Ashorn, Jaden } \\
\text { Bendabenda, Kenneth H. } \\
\text { Brown, Sonja Y. Hess, Emma } \\
\text { Kortekangas, Anna Lartey, } \\
\text { Kenneth Maleta, Brietta M. } \\
\text { Oaks, Eugenia Ocansey, } \\
\text { Harriet Okronipa, Jean Bosco } \\
\text { Ouedraogo, Anna Pulakka, Jer } \\
\text { ome W. Som, Christine P. } \\
\text { Stewart, Robert C. Stewart, } \\
\text { Stephen A. Vosti, Elizabeth } \\
\text { Yakes Jimenez, Kathryn G. } \\
\text { Dewey }\end{array}$ & 31 & 4205 & $\begin{array}{l}\text { Crianças com } 18 \text { meses de idade, que } \\
\text { participaram de testes conduzidos } \\
\text { como parte do Projeto Internacional } \\
\text { de Suplementos Nutricionais à Base } \\
\text { de Lípides (iLiNS)/ Gana, Malaui e } \\
\text { Burkina Faso }\end{array}$ & $\begin{array}{l}\text { Estudo } \\
\text { prospectivo de } \\
\text { coorte }\end{array}$ & $\begin{array}{l}\text { Seis fatores foram associados à linguagem e/ou } \\
\text { capacidade motora aos } 18 \text { meses de idade: crescimento } \\
\text { linear e ponderal da criança, variedade de materiais } \\
\text { lúdicos, atividades com cuidadores e diversidade } \\
\text { alimentar, e, status de hemoglobina/ferro da criança. }\end{array}$ & 7.035 \\
\hline $\begin{array}{l}\text { Prevalence and } \\
\text { socioeconomic } \\
\text { determinants of } \\
\text { development delay } \\
\text { among children in Ceará, } \\
\text { Brazil: A population- } \\
\text { based study }\end{array}$ & $\begin{array}{l}\text { Luciano Lima Correia, } \\
\text { Hermano Alexandre Lima } \\
\text { RochaID2, Christopher Robert } \\
\text { Sudfeld, Sabrina Gabriele } \\
\text { Maia Oliveira Rocha, Álvaro } \\
\text { Jorge Madeiro Leite, Jocileide } \\
\text { Sales Campos, Anamaria } \\
\text { Cavalcante e Silva }\end{array}$ & 03 & 3566 & $\begin{array}{l}\text { Crianças entre } 2 \text { e } 72 \text { meses de idade/ } \\
\text { Ceará, Brasil }\end{array}$ & $\begin{array}{l}\text { Estudo } \\
\text { transversal de } \\
\text { base } \\
\text { populacional }\end{array}$ & $\begin{array}{l}\text { A prevalência do atraso aumentou com a idade em } \\
\text { todos os domínios, e, os meninos apresentaram maior } \\
\text { risco de atraso na comunicação, no desenvolvimento } \\
\text { motor e pessoal-social em comparação com as } \\
\text { meninas. Observou-se associações robustas dos } \\
\text { indicadores de status socioeconômico e de risco para } \\
\text { atraso no desenvolvimento. }\end{array}$ & 2.740 \\
\hline $\begin{array}{l}\text { Risk and protective } \\
\text { factors for child } \\
\text { development: An } \\
\text { observational South } \\
\text { African birth cohort }\end{array}$ & $\begin{array}{l}\text { Kirsten Ann Donald, Catherine } \\
\text { J. WedderburnI, Whitney } \\
\text { Barnett, Raymond T. NhapiI, } \\
\text { Andrea M. RehmanI, Jacob A. } \\
\text { M. StadlerI, Nadia HoffmanI, } \\
\text { Nastassja Koen, Heather J. } \\
\text { ZarI, Dan J. Stein }\end{array}$ & 09 & 734 & $\begin{array}{l}\text { Gestantes entre } 20 \text { e } 28 \text { semanas de } \\
\text { gestação e seus respectivos filhos que } \\
\text { foram acompanhados desde o } \\
\text { nascimento até os } 2 \text { anos de idade/ } \\
\text { Cabo Ocidental, África do Sul }\end{array}$ & $\begin{array}{l}\text { Estudo de coorte } \\
\text { de base } \\
\text { populacional }\end{array}$ & $\begin{array}{l}\text { Houve evidências de que o sexo masculino } \\
\text { demonstrou maior vulnerabilidade a apresentar atraso } \\
\text { no desenvolvimento quando a criança teve baixo peso } \\
\text { ao nascer, foi exposta a anemia materna na gravidez e } \\
\text { condições socioeconômicas desfavoráveis. }\end{array}$ & 2.740 \\
\hline Trajectories of & Laudan B. Jahromi, Adriana J. & 09 & 204 & Filhos de mães adolescentes de & Estudo & Crianças com atraso/funcionalidade decrescente foram & 1.776 \\
\hline
\end{tabular}


Research, Society and Development, v. 10, n. 10, e411101018881, 2021

(CC BY 4.0) | ISSN 2525-3409 | DOI: http://dx.doi.org/10.33448/rsd-v10i10.18881

\begin{tabular}{|c|c|c|c|c|c|c|c|}
\hline $\begin{array}{l}\text { Developmental } \\
\text { Functioning Among } \\
\text { Children of Adolescent } \\
\text { Mothers: Factors } \\
\text { Associated With Risk for } \\
\text { Delay }\end{array}$ & $\begin{array}{l}\text { Umaña-Taylor, Kimberly A. } \\
\text { Updegraff, Katharine H. } \\
\text { Zeiders }\end{array}$ & & & $\begin{array}{l}\text { origem mexicana, acompanhados dos } \\
10 \text { aos } 41 \text { meses de idade/ México }\end{array}$ & longitudinal & $\begin{array}{l}\text { mais frequentes em famílias com renda mais baixa, } \\
\text { devido a menor disponibilidade de materiais didáticos } \\
\text { em casa, possuírem mães adolescentes com sintomas } \\
\text { depressivos e maior conflito parental com as figuras } \\
\text { maternas dos adolescentes. }\end{array}$ & \\
\hline $\begin{array}{l}\text { Neurodevelopment at } 2 \\
\text { years corrected age } \\
\text { among Vietnamese } \\
\text { preterm infants }\end{array}$ & $\begin{array}{l}\text { Thieu-Do, } \\
\text { Kruse,Wills, } \\
\text { Sabanathan, Clapham,Karup- } \\
\text { Pedersen, } \\
\text { Ngoc-Pham,Minh- Vu, } \\
\text { Landbo- Børresen }\end{array}$ & 4 & 184 & $\begin{array}{l}\text { Bebês nascidos com idade gestacional } \\
\text { menor que } 37 \text { semanas, e idade } \\
\text { corrigida de } 2 \text { anos/Vietnã }\end{array}$ & Estudo de coorte & $\begin{array}{l}\text { Os bebês pré-termos da UTI neonatal, pontuaram } \\
\text { significativamente menos na Bayley-III aos } 2 \text { anos, } \\
\text { comparados aos pares vietnamitas saudáveis nos } \\
\text { domínios: cognição, linguagem e motor. O ensino } \\
\text { materno superior apresentou associação positiva com } \\
\text { neurodesenvolvimento infantil. }\end{array}$ & 3.041 \\
\hline $\begin{array}{l}\text { Neurodevelopment of } \\
\text { HIV-exposed uninfected } \\
\text { children in South Africa: } \\
\text { outcomes from an } \\
\text { observational birth cohort } \\
\text { study }\end{array}$ & $\begin{array}{l}\text { Catherine J Wedderburn, } \\
\text { Shunmay Yeung, Andrea M } \\
\text { Rehman, Jacob A M Stadler, } \\
\text { Raymond T Nhapi, Whitney } \\
\text { Barnett, Landon Myer, } \\
\text { Diana M Gibb, Heather J Zar, } \\
\text { Dan J Stein, Kirsten A Donald }\end{array}$ & 7 & $\begin{array}{c}1065 \\
\text { (seguime } \\
\text { nto aos } 6 \\
\text { meses) } \\
1000 \\
\text { (seguime } \\
\text { ntos aos } \\
24 \\
\text { meses) }\end{array}$ & $\begin{array}{l}260 \text { bebês não infectados } \\
\text { ( } 61 \text { não infectados expostos ao HIV e } \\
199 \text { não expostos ao HIV) avaliados } \\
\text { aos } 6 \text { meses e } 732 \text { bebês (168 } \\
\text { expostos ao HIV não infectados e } 564 \\
\text { não expostos ao HIV) avaliados aos } \\
24 \text { meses/ Paarl, África do Sul }\end{array}$ & Estudo de coorte & $\begin{array}{l}\text { Para a linguagem receptiva e linguagem expressiva, as } \\
\text { proporções de crianças não infectadas e expostas ao } \\
\text { HIV com atraso no desenvolvimento foram maiores do } \\
\text { que as de crianças não expostas ao HIV. }\end{array}$ & 8.543 \\
\hline $\begin{array}{l}\text { Influencing factors of the } \\
\text { neurodevelopment of } \\
\text { high-risk infants }\end{array}$ & $\begin{array}{l}\text { Yuan Tian, } \\
\text { Chuncao Zhang, Guangjun } \\
\text { Yu,Xiangying Hu, } \\
\text { Zheng Pu, Liyu Ma }\end{array}$ & 1 & 484 & $\begin{array}{l}\text { Lactentes acompanhados do } \\
\text { nascimento aos } 9 \text { meses de idade, que } \\
\text { atenderam aos critérios para } \\
\text { classificação de alto risco/ Xangai, } \\
\text { China }\end{array}$ & $\begin{array}{l}\text { Análise } \\
\text { retrospectiva de } \\
\text { dados coletados }\end{array}$ & $\begin{array}{l}\text { A incidência de anormalidades no } \\
\text { neurodesenvolvimento de bebês de alto risco com } \\
\text { menos de } 1 \text { ano é alto. O nascimento prematuro e os } \\
\text { maus hábitos dos pais são fatores significativos que } \\
\text { afetam o neurodesenvolvimento. Além disso, o } \\
\text { monitoramento e as intervenções precoces ajudam a } \\
\text { melhorar o neurodesenvolvimento de bebês de alto } \\
\text { risco. }\end{array}$ & 2.157 \\
\hline $\begin{array}{l}\text { Early psychomotor } \\
\text { development of low-risk } \\
\text { preterm infants: Influence } \\
\text { of gestational age and } \\
\text { gender }\end{array}$ & $\begin{array}{l}\text { Domenico M. Romeo,Claudia } \\
\text { Brogna, Francesco Sini,Mario } \\
\text { G. Romeo, Francesco Cota, } \\
\text { Daniela Ricci }\end{array}$ & 14 & 188 & $\begin{array}{l}\text { Bebês prematuros acompanhados dos } \\
3 \text { meses aos } 2 \text { anos de idade, } \\
\text { subdivididos em } 2 \text { grupos de acordo } \\
\text { com a idade gestacional (IG): muito } \\
\text { pré-termo ( } 32 \text { semanas de IG) e pré- } \\
\text { termo tardio (entre } 33-36 \text { semanas de } \\
\text { IG) e um grupo controle de bebês } \\
\text { com IG de } 37-42 \text { semanas, e peso ao } \\
\text { nascer igual ou superior a } 2550 \text { g, sem } \\
\text { histórico de cuidados pré-natais, } \\
\text { perinatais ou pós-natais significativos/ }\end{array}$ & $\begin{array}{l}\text { Investigação } \\
\text { prospectiva de } \\
\text { seguimento }\end{array}$ & $\begin{array}{l}\text { Os principais resultados indicam que tanto os } \\
\text { prematuros extremos quanto os tardios apresentaram } \\
\text { escores globais significativamente menores do que os } \\
\text { bebês nascidos a termo em cada avaliação no HINE, } \\
\text { aos } 3 \text { meses para as subseções "nervo craniano" e } \\
\text { "postura", e, em todas as idades para "tônus"; nenhuma } \\
\text { diferença significativa foi evidenciada em } \\
\text { performances neurológicas. No MDI, os pré-termos } \\
\text { extremos apresentaram escores inferiores } \\
\text { significativos dos apresentados pelos prematuros } \\
\text { tardios quanto os nascidos a termo. }\end{array}$ & 2.510 \\
\hline
\end{tabular}


Research, Society and Development, v. 10, n. 10, e411101018881, 2021

(CC BY 4.0) | ISSN 2525-3409 | DOI: http://dx.doi.org/10.33448/rsd-v10i10.18881

\begin{tabular}{|c|c|c|c|c|c|c|c|}
\hline & & & & Catânia, Itália & & & \\
\hline $\begin{array}{l}\text { Maternal lifestyle during } \\
\text { pregnancy and child } \\
\text { psychomotor } \\
\text { development — Polish } \\
\text { Mother and Child Cohort } \\
\text { study }\end{array}$ & $\begin{array}{l}\text { Kinga Polańska, Paweł } \\
\text { Muszyńskia, Wojciech } \\
\text { Sobalaa, Emila Dziewirskaa, } \\
\text { Dorota Merecz-Kotb, } \\
\text { Wojciech Hankea }\end{array}$ & 31 & $\begin{array}{c}538 \\
\text { lactantes }\end{array}$ & $\begin{array}{l}\text { Mulheres durante o primeiro trimestre } \\
\text { de gravidez para acompanhar os } \\
\text { fatores relacionados ao estilo de vida } \\
\text { materno, que foram avaliadas antes e } \\
\text { durante a gravidez, e seus respectivos } \\
\text { filos, que foram avaliados no primeiro } \\
\text { e no segundo ano de vida/ Polônia }\end{array}$ & Estudo de coorte & $\begin{array}{l}\text { Observou-se associação significativa entre a exposição } \\
\text { pré-natal aos constituintes do tabaco e a diminuição do } \\
\text { desenvolvimento motor infantil em avaliações } \\
\text { realizadas no primeiro e no segundo ano de vida. O } \\
\text { baixo peso materno pré-gravidez esteve associado à } \\
\text { diminuição das habilidades linguísticas aos } 12 \text { meses } \\
\text { de idade e desenvolvimento cognitivo e motor aos } 24 \\
\text { meses de idade do lactente. A prática de atividade } \\
\text { física moderada por pelo menos } 30 \text { minutos diários } \\
\text { durante a gravidez foi benéfica para o } \\
\text { desenvolvimento da linguagem infantil aos dois anos } \\
\text { de idade. }\end{array}$ & 1.969 \\
\hline $\begin{array}{l}\text { Maternal and partner } \\
\text { prenatal alcohol use and } \\
\text { infant cognitive } \\
\text { development }\end{array}$ & $\begin{array}{l}\text { Clare Delyse Hutchinsonac de } \\
\text { Lucy, Burnsa George } \\
\text { Youssefcd, Judy Wilsona, } \\
\text { Elizabeth Elliottf, Steve } \\
\text { Allsopg, Jake Najmanh, Sue } \\
\text { Jacobsi, Larissa Rossena, } \\
\text { Craig Olssonc de Richard } \\
\text { Matticka }\end{array}$ & 9 & 1331 & $\begin{array}{l}\text { Mulheres grávidas ( } \mathrm{n}=1331 \text { ) e seus } \\
\text { parceiros }(\mathrm{n}=699) \text { foram recrutados } \\
\text { em clínicas pré-natais em três } \\
\text { hospitais públicos metropolitanos na } \\
\text { Austrália e completaram entrevistas } \\
\text { detalhadas sobre o uso de álcool } \\
\text { durante a gravidez. Os bebês foram } \\
\text { avaliados aos } 12 \text { meses de idade/ } \\
\text { Austrália }\end{array}$ & Estudo de coorte & $\begin{array}{l}\text { Foram observadas evidências de capacidade cognitiva } \\
\text { prejudicada nos lactentes, associada ao uso de álcool } \\
\text { pelas mães ou seus parceiros. }\end{array}$ & 3.951 \\
\hline
\end{tabular}

Fonte: Autores. 
Os dados demonstram que os estudos mais citados até 2020 foram: Silva, Engstron e Miranda (2015) com 38 citações, Polańska et al. (2015) e Prado et al. (2017) com 31 citações cada um. Em relação ao número de participantes, o estudo com maior amostra foi o de revisão sistemática, a qual revisou estudos relacionados à influência da classificação socioeconômica sobre as habilidades motoras grossas em crianças em idade escolar, sendo observado que, quanto maior a classificação socioeconômica, maior a pontuação alcançada nas avaliações de habilidades motoras. Com relação ao tipo de participantes, observou-se a presença de fatores de risco biológico (prematuros, baixo peso ao nascimento, doenças congênitas) e socioeconômico (escolaridade materna, baixa renda, dificuldade para acesso à intervenção), e a faixa etária abordada, foi de recém-nascidos a seis anos de idade. Quanto ao delineamento de estudo, o mais frequente foi o transversal, em 17 estudos, seguido pelo estudo de coorte em 10 artigos, sendo ambos com análise quantitativa. Os resultados dos estudos selecionados, indicaram a baixa escolaridade materna, o baixo nível socioeconômico familiar, e, pouca disponibilidade de recursos para estimulação da criança, como fatores mais frequentes para o atraso no desenvolvimento neurossensoriomotor.

Trinta e uma revistas apresentaram artigos publicados acerca do tema do estudo, e, as com mais artigos publicados foram: Journal of Human Growth and Development (8\%), The Journal of Physical Education (5\%), Trends in Psychiatry and Psychotherapy (5\%), BMC Pediatrics (5\%) e PLOS ONE (5\%). Não foi encontrado o fator de impacto de 20 (64\%) periódicos (Quadro 1).

Quadro 1. Número de artigos publicados e fator de impacto do periódico.

\begin{tabular}{|c|c|c|}
\hline Periódicos & $\begin{array}{c}\text { Número de } \\
\text { artigos }\end{array}$ & $\begin{array}{l}\text { Fator de } \\
\text { impacto }\end{array}$ \\
\hline BMC Pediatrics & 2 & 1.909 \\
\hline Jornal de Pediatria & 1 & 2.029 \\
\hline Journal of Child Psychology and Psychiatry & 1 & 7.035 \\
\hline PLOS ONE & 2 & 2.740 \\
\hline American Journal on Intellectual and Developmental Disabilities & 1 & 1.776 \\
\hline Archives of Disease in Childhood & 1 & 3.041 \\
\hline Lancet Child \& Adolescent Health & 1 & 8.543 \\
\hline General Psychiatry & 1 & 2.157 \\
\hline European Journal of Paediatric Neurology & 1 & 2.510 \\
\hline Early Human Development & 1 & 1.969 \\
\hline Drug and Alcohol Dependence & 1 & 3.951 \\
\hline Revista CEFAC & 1 & - \\
\hline Fisioterapia e Pesquisa & 2 & - \\
\hline The Journal of Physical Education & 2 & - \\
\hline Trends in Psychiatry and Psychotherapy & 1 & - \\
\hline Fisioterapia Brasil & 1 & - \\
\hline Journal of Human Growth and Development & 3 & - \\
\hline International Journal of Cardiovascular Sciences & 1 & - \\
\hline Revista Brasileira de Terapia Intensiva & 1 & - \\
\hline Revista subjetividade & 1 & - \\
\hline Brazilian Journal of Cardiovascular Surgery's & 1 & - \\
\hline Revista Medicina (Ribeirão Preto) & 1 & - \\
\hline Cadernos de Saúde Pública & 1 & - \\
\hline Estudos e pesquisas em psicologia & 1 & - \\
\hline Pensando Famílias & 1 & - \\
\hline Revista Paulista de Pediatria & 1 & - \\
\hline Archivos Argentinos de Pediatría & 1 & - \\
\hline Revista Panamericana de Salud Pública & 1 & - \\
\hline Revista Chilena de Pediatría & 1 & - \\
\hline British Medical Journal Open & 1 & - \\
\hline Psychological Trauma: Theory, Research, Practice, and Policy & 1 & - \\
\hline
\end{tabular}

Fonte: Autores. 
Quanto ao número de artigos publicados sobre o tema, por ano, observou-se que em 2016 houve um maior número de publicações, com declínio nos anos seguintes (Gráfico 1).

Gráfico 1. Número de artigos publicados entre os anos de 2015 e 2020.

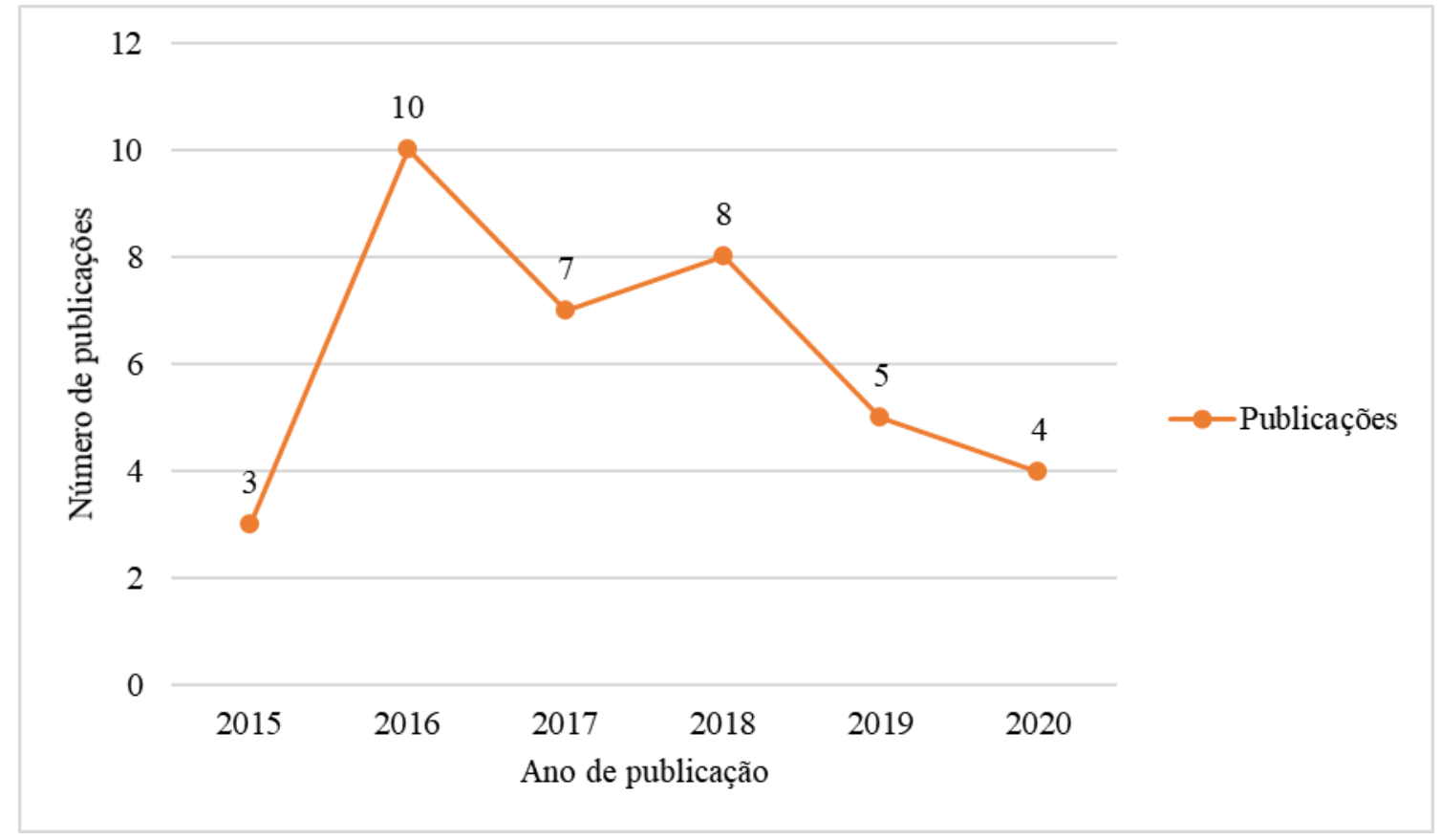

Fonte: Autores.

Dentre os países com maior número de artigos publicados sobre o tema, entre 2015 e 2020, o Brasil apresentou-se em primeiro lugar, com 20 estudos publicados, seguido por África do Sul com 4, China com 2, e, 7 em outros países.

Na Figura 2, é possível observar as palavras citadas com maior frequência, dentre os descritos presentes nos artigos selecionados. 
Figura 2. Frequência das palavras observadas dentre os descritores dos artigos incluídos.

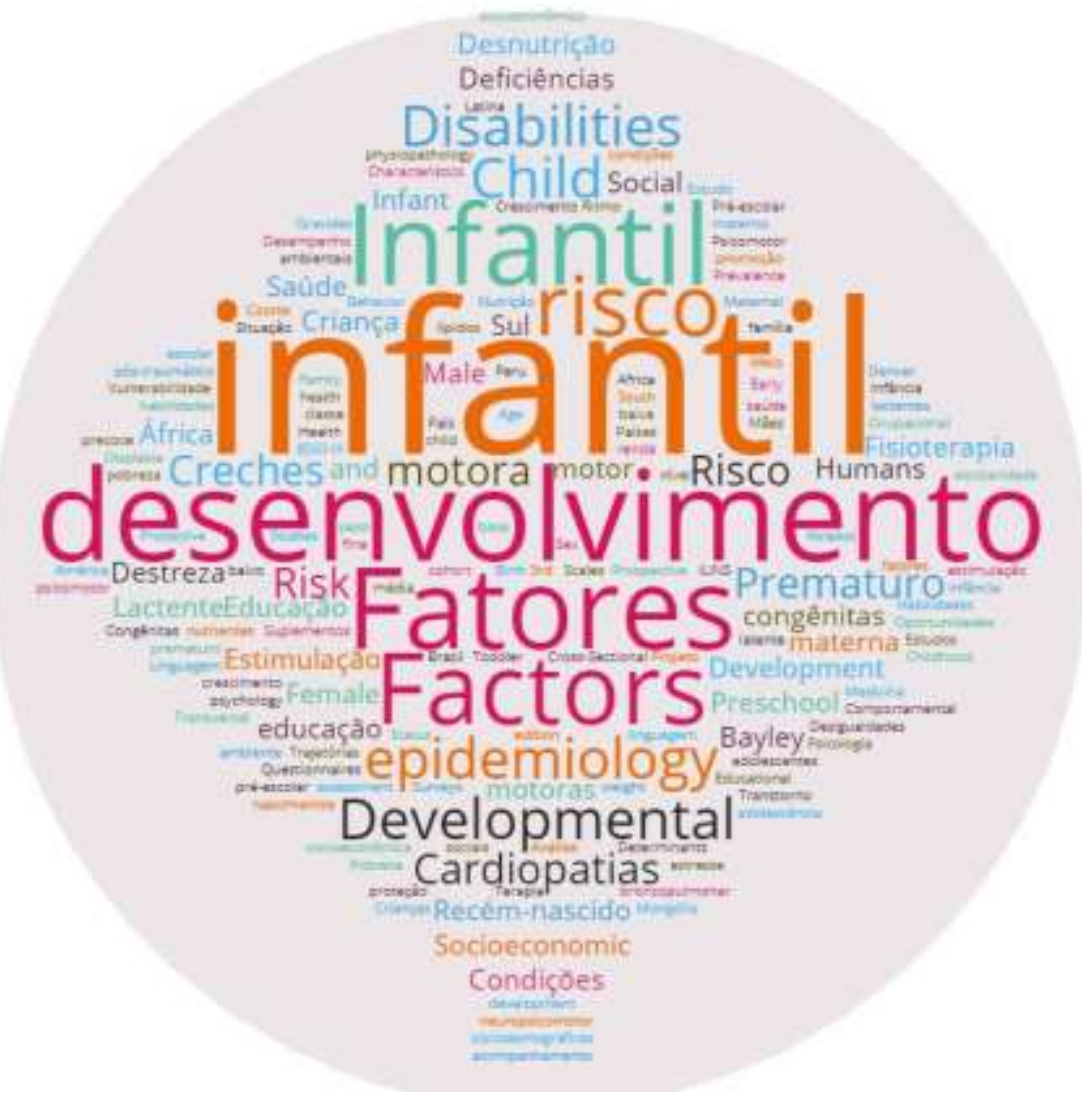

Fonte: Autores.

Entre os descritores dos artigos selecionados os mais frequentes foram infantil, desenvolvimento e fatores de risco, respectivamente. As demais palavras foram menos citadas, e não, em todos os artigos selecionados.

\section{Discussão}

Considerando o objetivo do presente estudo, em mapear o estado da arte, ou seja, o rigor metodológico da produção científica atual, sobre os fatores de risco para atraso no desenvolvimento infantil, foi possível observar uma escassez de publicações. Apenas 13 estudos foram publicados em periódicos com classificação de fator de impacto. Tal fato pode estar relacionado, com a qualidade metodológica das publicações realizadas acerca do tema. Com base nessa premissa, é importante que se desenvolvam mais estudos sobre a temática, com rigor metodológico, para poder alcançar maior visibilidade científica. Contudo, cabe destacar, que nem sempre um estudo publicado em periódico sem fator de impacto internacional, é metodologicamente ruim ou inadequado, pois existem periódicos ainda sem classificação, mas com publicações de alta qualidade.

Reforçando a necessidade de mais estudos, observou-se que, desde o ano de 2018, o número de publicações sobre o tema tem sofrido declínio progressivo. Este achado, contrapõe a tendência mundial de crescimento do número de publicações científicas com o passar dos anos, e o aumento do uso de base de dados online. De acordo com Souza (2006), em 1981 foram publicados 440.286 artigos científicos no mundo inteiro, e, em 2002 aumentou para 872.018, demonstrando um aumento expressivo da quantidade de artigos científicos publicados no decorrer dos anos, e, o avanço das bases de dados online. Ademais, o declínio progressivo do número de publicações sobre o tema em questão, também limita a implementação do modelo biopsicossocial com base nos avanços na área da saúde. 
O modelo biopsicossocial trata-se de compreender a saúde, como completo bem-estar físico, social e mental, apontando para a necessidade de os profissionais da área da saúde serem capazes de identificar vulnerabilidades biológicas e ambientais no indivíduo. Para tal compreensão, são necessários estudos que indiquem os fatores intrínsecos e extrínsecos que constituem riscos de atrasos no desenvolvimento, influenciando a saúde do indivíduo (Pereira, Barros, \& Augusto, 2011).

Entre 2015 e 2020, o Brasil foi o país com maior número de estudos desenvolvidos sobre o tema, dado este relevante, se considerarmos a grande desigualdade social, a qual expõe a população infantil à fatores de risco, em especial, fatores ambientais (Campello, Gentili, Rodrigues, \& Hoewell, 2018). Tal fato, reforça a importância de estudos sobre o tema, sendo relevante para elaboração de políticas públicas, que permitam a identificação e a intervenção nas alterações do desenvolvimento neuropsicomotor infantil.

É importante destacar, que para a intervenção ser considerada precoce, ela deve ser realizada no período de maior plasticidade cerebral do bebê, sendo os primeiros quatro meses de idade a época ideal para iniciar, a fim de se intervir antes que padrões inadequados de movimento, e, posturas atípicas tenham sido apresentados (Formiga, \& Ramos, 2016). Além disso, a detecção de fatores de risco, preferencialmente, antes do terceiro ano de idade, favorece o início da estimulação, permitindo que a criança alcance potencial desenvolvimento (Hallal, Marques, \& Braccialli, 2008).

Quanto ao número de publicações com fator de impacto, esperava-se mais, considerando a importância e a necessidade de qualidade da informação transmitida ao leitor, por meio de ferramentas de avaliação confiáveis (Ferreira, \& Krzyzanowski, 2003). Neste sentido, a principal ferramenta utilizada para catalogar os índices bibliométricos, referentes as publicações da literatura científica produzida anualmente, é o banco de dados produzido pelo Institute for Scientific Information (ISI), o qual utiliza dois critérios, para escolher as revistas que incorporam seu banco de dados, sendo estes, a periodicidade, e, o fator de impacto, que é medido pelo número de citações dos artigos. O Journal of Citation Reports (JCR) reúne os dados informados pelo ISI, a fim de revelar o número de citações dos artigos publicados em determinado ano, e, calcular o fator de impacto da revista avaliada (Pinto, \& Andrade, 1999). O objetivo principal da utilização do fator de impacto, é avaliar a produção científica dos autores e a qualidade das publicações, por meio de um cálculo, o qual considera: o número de citações dos artigos de uma revista em todas as revistas inseridas no ISI, o número de publicações realizados pela mesma revista nos dois anos anteriores, e, por meio deste cálculo, classificam-se os periódicos científicos inseridos no JCR do ISI (Ruiz, Greco, \& Braile, 2009).

Vale ressaltar, que dos 31 periódicos com estudos selecionados, 20 não tinha classificação pelo JCR, o que se torna um achado preocupante, por demonstrar que vários dos estudos pré-selecionados, podem não atender uma avaliação padronizada de qualidade, ou ainda, serem desperdiçados, não alcançando um público maior, devido estar publicado em revistas de baixo ou sem fator de impacto.

Em relação ao delineamento do estudo, o mais frequente foi o transversal, analisando a exposição a um fator ou causa, no exato momento, ou intervalo de tempo analisado. Este delineamento de estudo é relevante na avaliação dos efeitos causados por fatores imutáveis do indivíduo, sendo, portanto, indicado para avaliar a influência de fatores de riscos biológicos, porém, não sendo a melhor opção para avaliar a influência de fatores de risco socioeconômicos, pois, os mesmos podem sofrer inúmeras modificações com o decorrer do tempo (Hochman, Nahas, Oliveira, \& Ferreira, 2005).

Dentre os fatores de risco socioeconômicos mais citados, para possível atraso no desenvolvimento neuromotor, encontrou-se a baixa escolaridade materna, o baixo nível socioeconômico familiar e a pouca disponibilidade de recursos para a estimulação da criança. Estes dados corroboram com o estudo de Maria-Mengel e Linhares (2007), no qual concluiu-se que os fatores de risco psicossociais e nutricionais merecem maior atenção por aumentar a possibilidade de problemas de desenvolvimento, sendo importante considerar, de forma conjunta, tanto os fatores de risco como os recursos que podem influenciar no desenvolvimento. Desta forma, a condição socioeconômica da família constitui-se um fator de risco relevante 
para o desenvolvimento neuromotor infantil. Esta relaciona-se diretamente com o grau de escolaridade do cuidador, as condições de habitação em áreas de risco, e, a disponibilidade de recursos para estimulação da criança.

Vale ressaltar que o Brasil apresentou o maior número de estudos sobre o tema no período de cinco anos, o que é um ponto positivo, considerando a grande desigualdade social presente no país, possibilitando a elaboração de políticas e estratégias do Sistema Único de Saúde (SUS), visando assegurar que as crianças se desenvolvam de forma integral e com qualidade de vida.

Assim, a relevância do presente estudo, pode ser observada à medida que demonstra a qualidade das publicações sobre fatores de risco para o desenvolvimento neuromotor, buscando incentivar a produção de mais artigos, observando os critérios e rigor metodológicos exigidos se publicar em periódicos de qualidade comprovada pelo JCR, garantindo maior confiabilidade dos achados, e, maior alcance profissional de nível técnico-científico. Ademais, pode contribuir, para o embasamento e criação de políticas públicas, que visem proporcionar um melhor desenvolvimento infantil e de forma integral.

\section{Conclusão}

Por meio desta revisão, concluiu-se que fatores biológicos e socioeconômicos estão frequentemente relacionados ao atraso no desenvolvimento neuromotor da criança. Os fatores mais observados nos estudos foram os socioeconômicos, relacionados a baixa escolaridade materna, a baixa renda familiar e a pouca disponibilidade de recursos para estimulação da criança. Vale ressaltar, que para elaboração de políticas públicas eficazes, buscando proporcionar o desenvolvimento infantil de forma integral, é fundamental que haja o embasamento técnico-científico sobre os fatores de risco que interferem nesse processo, indicando, portanto, a relevância de estudos acerca deste tema.

Os resultados demonstram, ainda, um declínio na produção científica acerca do tema com o passar dos anos. Isto indica a necessidade de mais estudos sobre o tema, e, que os mesmos sejam realizados, com rígido critério metodológico, para que possam ser publicados em periódicos com bom fator de impacto, alcançando um maior público, e, possibilitando a elaboração de políticas públicas de saúde.

\section{Referências}

Alves, C. O., Magalhães, L. C., Moreira, R. S., Silveira, I. F., Machado, M. G. P., \& Viana, C. F. B. (2016). Factors associated with the development of preterm children at four and eight months of corrected gestational age. Journal Of Human Growth And Development, 26 (1), $42-48$.

Araujo, L. B., Mélo, T. R., Israel, \& V. L. (2017). Low birth weight, family income and paternal absence as risk factors in neuropsychomotor development. Journal Of Human Growth And Development, 27 (3), 272-280.

Bedregal, P.,Hernández, V., Mingo, M. V., Castañón, C., Valenzuela, P., Moore, R., Lacruz, R. De., \& Castro, D. (2016). Desigualdades en desarrollo infantil temprano entre prestadores públicos y privados de salud y factores asociados en la Región Metropolitana de Chile. Revista Chilena de Pediatría, 87 (5), 351358 .

Blakstad, M. M., Smith, E. R., Etheredge, A., Locks, L. M., Mcdonald, C. M., Kupka, R., Kisenge, R., Aboud, S., Bellinger, D., \& Sudfeld, C. R. (2019). Nutritional, Socioeconomic, and Delivery Characteristics Are Associated with Neurodevelopment in Tanzanian Children. The Journal Of Pediatrics, 207, 7179.

Campello, T., Gentili, P., Rodrigues, M., \& Hoewell, G. R. (2018). Faces da desigualdade no Brasil: um olhar sobre os que ficam para trás. Saúde Debate, 42 (3), 54-66.

Candéa, G. B., Silva, J. G., Candéa, A. L. P., Vidal, J. M. De., \& Telles, S. C. C. De. (2017). A influência do status socioeconômico sobre as habilidades motoras grossas nas crianças em idade escolar. Fisioterapia Brasil, 18 (6), 757-766.

Chueke, G. V., \& Amatucci, M. (2015). O que é bibliometria? Uma introdução ao Fórum. Internext, 10(2), 01-05.

Correia, L. L., Rocha, H. A. L., Sudfeld, C. R., Rocha, S. G. M O., Leite, A. J. M., Campos, J. S., \& Silva, A. C. (2019). Prevalence and socioeconomic determinants of development delay among children in Ceará, Brazil: a population-based study. Plos One, 14 (11), e0215343.

Corsi, C., Santos, M. M. Dos., Marques, L. A. P. De., \& Rocha, N. A. C. F. (2016). Impact of extrinsic factors on fine motor performance of children attending day care. Revista Paulista de Pediatria, 34 (4), 439-446. 
Dagvadorj, A., Ganbaatar, D., Balogun, O. O., Yonemoto, N., Bavuusuren, B., Takehara. K., Mori, R., \& Akahira-Azuma, M. (2018). Maternal sociodemographic and psychological predictors for risk of developmental delays among young children in Mongolia. Bmc Pediatrics, 18 (1), 68-76.

Delgado, D. A., Michelon, R. C., Gerszon, L. R., Almeida, C. S., \& Alexandre, M. G. Da. (2020). Avaliação do desenvolvimento motor infantil e sua associação com a vulnerabilidade social. Fisioterapia e Pesquisa, 27 (1), 48-56.

Díaz A. A., Bacallao G. J., Vargas-Machuca, R., \& Aguilar V. R. (2017). Desarrollo infantil en zonas pobres de Perú. Rev Panam Salud Publica, 41, 71-79.

Do, C. H. T., Kruse, A. Y., Wills, B., Sabanathan, S., Clapham, H., Pedersen, F. K., Pham, T. N., Vu, P. M. V., \&Borresen, M. L. (2020). Neurodevelopment at 2 years corrected age among Vietnamese preterm infants. Arch. Dis. Child. 105, 134-140.

Donald, K. A., Wedderburn, C. J., Barnett, W., Nhapi, R. T., Rehman, A. M., Stadler, J. A. M., Hoffman, N., Koen, N., Zar, H. J., \& Stein, D. J. (2019). Risk and protective factors for child development: an observational south african birth cohort. Plos Medicine, 16 (9), e1002920.

Duarte, M. G., Duarte, G. S. D., Nobre, G. C., Bandeira, P. F. R., Santos, J. O. L. Dos., \& Barros, J. L. C. Da. (2016). Desenvolvimento motor e fatores associados de crianças entre 36 e 42 meses em um contexto do baixo amazonas. Journal Of Physical Education, 27 (1), 1-10.

Ferreira, M. C. G., \& Krzyzanowski, R. F. (2003). Periódicos científicos: critérios de qualidade. Pesquisa Odontológica Brasileira, 17 (1), $43-48$.

Formiga, C. K. M. R., \& Ramos, B. A. (2017). Programas de intervenção precoce: orientações gerais e experiências. Revista Diálogos e Perspectivas em Educação Especial, 3 (2), 111-116.

Hallal, C. Z., Marques, N. R., \& Braccialli, L. M. P. (2008). Aquisição de habilidades funcionais na área de mobilidade em crianças atendidas em um programa de estimulação precoce. Journal Of Human Growth And Development, 18 (1), 27-34.

Hochman, B., Nahas, F. X., Oliveira, R. S. De., \& Ferreira, L. M. (2005). Desenhos de pesquisa. Acta Cirurgica Brasileira, 20 (2), $2-9$.

Jahromi, L. B., Umaña-Taylor, A. J., \& Updegraff, K. A., Zeiders, K. H. (2016). Trajectories of Developmental Functioning Among Children of Adolescent Mothers: factors associated with risk for delay. American Journal On Intellectual And Developmental Disabilities, 121 (4), $346-363$.

Koen, N., Brittain, K., Donald, K. A., Barnett, W., Koopowitz, S., Maré, K., Zar, H. J., \& Stein, D. J. (2017). Maternal posttraumatic stress disorder and infant developmental outcomes in a South African birth cohort study. Psychological Trauma: Theory, Research, Practice, and Policy, 9 (3), 292-300.

Leal, L. S., Siva, R. L. M. Da., Aita, K. M. S. C., Monteiro, R. P. A. De., \& Montalvão, T. C. De. (2016). Assessment of Motor Development of Children with Congenital Heart Disease. International Journal Of Cardiovascular Sciences, 29 (2), 103-109.

Lejarraga, H., Kelmansky, D. M., \& Nunes, F. (2018). Developmental tempo in children aged 0-5 years living under unfavorable environmental conditions. Arch Argent Pediatr, 116 (2), 210-215.

Mari, M. A., Cascudo, M. M., \& Alchieri, J. C. (2016). Congenital Heart Disease and Impacts on Child Development. Brazilian Journal Of Cardiovascular Surgery, 31 (1), 31-37.

Maria-Mengel, M. R. S., \& Linhares, M. B. M. (2007). Risk factors for infant developmental problems. Revista Latino-Americana de Enfermagem, 15, 837842.

Martins, T. S. A., Vilela, R. V., Pereira, F. V., \& Cária, N. Z. \& Faria, H. P. (2013). Implementation of the evaluation of growth and psychomotor development in children under 5 years in the Grajau family health unit of the city of Brumadinho - MG, by the UFMG internship in rural areas. Revista Médica de Minas Gerais, 23 (1), 27-32.

Matos, L. A., Cavalcante, L. I. C., \& Costa, E. F. (2017). Características do Ambiente Sociofamiliar e Desenvolvimento Neuropsicomotor de Crianças: associações e implicações. Revista Subjetividades, 16 (3), 97-108.

Mccormack, C., Hutchinson, D., Burns, L., Youssef, G., Wilson, J., Elliott, E., Allsop, S., Najman, J., Jacobs, S., Rossen, L., Olsson, C., \& Mattick, R. (2018). Maternal and partner prenatal alcohol use and infant cognitive development. Drug and Alcohol Dependence, 185, $330-338$.

Mezzari, S. S., Donadio, M. V. F., Gerzson, L. R., \& Almeida, C. S. De. (2019). Desenvolvimento neuropsicomotor e desnutrição de uma população de risco de um bairro de Porto Alegre. Medicina (Ribeirao Preto. Online), 52 (2), 80-90.

Oliveira, C. C. L., Silva, R., Freitas, I., Gomes, M., \& Cândida, M. (2016). Factors associated with the development of preterm children at four and eight months of corrected gestational age. J Hum Growth Dev, 26 (1), 42-48.

Paula, I. R., Oliveira, J. C. S., Batista, A. C. F., Nascimento, L. C. S., Araújo, L. B. De., Ferreira, M. B., Gomes, M. B., \& Azevedo, V. M. G. O. De. (2020). Influência da cardiopatia congênita no desenvolvimento neuropsicomotor de lactentes. Fisioterapia e Pesquisa, 27 (1), $41-47$.

Pereira, K., Valentini, N. C., \& Saccani, R. (2016). Brazilian infant motor and cognitive development: longitudinal influence of risk factors. Pediatrics International, 58 (12), 1297-1306.

Pereira, T. T. S. O., Barros, M. N. S. Dos., \& Augusto, M. C. N. A. De. (2011). O Cuidado em Saúde: o Paradigma Biopsicossocial e a Subjetividade em Foco. Mental: Revista de saúde mental e subjetividade da UNIPAC, 9 (17), 523-536.

Pereira, V. A., Silva-Marinho, C. S. O., Rolim, O. M. P., Chiodelli, T., \& Donatto, M. L. (2015) Investigação de Fatores Considerados de Risco para o Desenvolvimento Motor de Lactentes até o Terceiro Mês. Pensando Famílias, 19 (2), 73-85.

Pinto, A. C., \& Andrade, J. B. De. (1999). Fator de impacto de revistas científicas: qual o significado deste parâmetro? Química Nova, 22 (3),448-453.

Polanska, K., Muszynski, P., Sobala, W., Dziewirska, E., Merecz-kot, D., \& Hanke, W. (2015). Maternal lifestyle during pregnancy and child psychomotor development - Polish Mother and Child Cohort study. Early Human Development, 91 (5), 317-325. 
Prado, E. L., Abbeddou, S., Adu-Afarwuah, S., Arimond, M., Ashorn, P., Ashorn, U., Bendabenda, J., Brown, K. H., Hess, S. Y., \& Kortekangas, E. (2017). Predictors and pathways of language and motor development in four prospective cohorts of young children in Ghana, Malawi, and Burkina Faso. Journal Of Child Psychology And Psychiatry, 58 (11),1264-1275.

Queiroz, D. R. Da., Guimarães, T. G. M., Campos, C. M. C., Feitoza, A. H. P., Henrique, R. S. Dos., Santos, M. A. M. Dos., \& Cattuzzo, M. T. (2020). Effect of school type and family income on motor competence changes in pre-school children: a repeated cross sectional study. Journal Of Physical Education, 31 (1), 3118-3126.

Ramdin, T., Ballot, D., Rakotsoane, D., Madzudzo, L., Brown, N., Chirwa, T., Cooper, P., \& Davies, V. (2018). Neurodevelopmental outcome of late preterm infants in Johannesburg, South Africa. Bmc Pediatrics, 18 (1), 326-332.

Romeo, D. M., Brogna, C., Sini, F., Romeo, M. G., Cota, F., \& Ricci, D. (2016). Early psychomotor development of low-risk preterm infants: influence of gestational age and gender. European Journal Of Paediatric Neurology, 20 (4), 518-523.

Ruiz, M. A., Greco, O. T., \& Braile, D. M. (2009). Fator de impacto: Importância e influência no meio editorial, acadêmico e científico. Revista Brasileira de Hematologia e Hemoterapia.

Santos, A. P. M. Dos., Villaverde, L. N., Costa, A. N. F., Santos, M. O. De., Gregório, E. C., Andreis, L. M., \& Rosa, F. (2016). Biopsychosocial factors contributing to delayed motor development in children: a longitudinal study. Journal Of Human Growth And Development, 26 (1), 112-118.

Silva, A. C. D. Da., Engstron, E. M., \& Miranda, C. T. De. (2015). Fatores associados ao desenvolvimento neuropsicomotor em crianças de 6-18 meses de vida inseridas em creches públicas do Município de João Pessoa, Paraíba, Brasil. Cadernos de Saúde Pública, 31 (9), 1881-1893.

Silva, L. V., Araújo, L. B., \& Azevedo, V. M. G. O. De. (2018). Avaliação do desenvolvimento neuropsicomotor de lactentes nascidos prematuros com e sem displasia broncopulmonar no primeiro ano de vida. Revista Brasileira de Terapia Intensiva, 30 (2), 174-180.

Silva, M. L. Da., Cavalcante, L. I. C., Heumann, S., \& Lima, T. V. R. (2018). Relação entre gênero e desempenho neuropsicomotor de crianças em Belém, Brasil. Ciência \& Saúde Coletiva, 23 (8), 2721-2730.

Souza, E. P. S. De. (2006). Publicação de revistas científicas na Internet. Brazilian Journal of Cardiovascular Surgery, 21 (1), $24-28$.

Souza, M. T., Silva, M. D., \& Carvalho, R. (2010). Integrative review: what is it? How to do it? Einstein (São Paulo), 8 (1), 102-106.

Souza, J. M De., \& Veríssimo, M. L. Ó. R. De. (2015). Child development: analysis of a new concept. Revista Latino-Americana de Enfermagem, 23 (6), 1097-1104.

Teixeira, M. C. T. V., Alckmin-Carvalho, F., Emerich, D. R., Cevallos, P. V., \& Paula, C. S. (2017). Indicadores de atraso no desenvolvimento em crianças de creche advindas de famílias de baixa renda. Estudos e Pesquisa em Psicologia, 17 (3), 1042-1062.

Tella, P., Piccolo, L. R. Da., Rangel, M. L., Rohde, L. A., Polanczyk, G. V., Miguel, E. C., Grisi, S. J. F. E., Fleitlich-Bilyk, B., \& Ferraro, A. A. (2018). Socioeconomic diversities and infant development at 6 to 9 months in a poverty area of São Paulo, Brazil. Trends in Psychiatry and Psychotherapy, 40 (3), 232-240.

Tian, Y. Zhang, C., Yu, G. Hu, X., Pu, Z., \& Ma, L. (2018). Influencing factors of the neurodevelopment of high-risk infants. General Psychiatry, 31, e100034.

Torquato, I. M. B., Forte, F. D. S., França, J. R F. S, Silva, M. F. O. C., \& Reichert, A. P. S. (2019). Efetividade de uma intervenção com mães para a estimulação de crianças menores de dois anos. Revista Latino-Americana de Enfermagem, 27, e3216.

Wedderburn, C. J., Yeung, S., Rehman, A. M., Stadler, J. A. M., Nhapi, R. T., Barnett, W., Myer, L., Gibb, D. M., Zar, H. J., \& Stein, D. J. (2019). Neurodevelopment of HIV-exposed uninfected children in South Africa: outcomes from an observational birth cohort study. The Lancet Child \& Adolescent Health, 3 (11), 803-813.

Zago, J. T. C. De., Pinto, P. A. F., Leite, H. R., Santos, J. N., \& Morais, R. L. S. De. (2017). Associação entre o desenvolvimento neuropsicomotor e fatores de risco biológico e ambientais em crianças na primeira infância. Revista Cefac, 19 (3), 320-329.

Zhang, J., Guo, S., Li, Y., Wei, Q., Zhang, C., Wang, X., Luo, S., Zhao, C., \& Scherpbier, R. W. (2018). Factors influencing developmental delay among young children in poor rural China: a latent variable approach. Bmj Open, 8 (8), 021628. 